\title{
Boundary layer flow and heat transfer past a shrinking sheet in a copper-water nanofluid
}

\begin{abstract}
The problem of laminar fluid flow which results from the shrinking of a flat surface in a water-based copper $(\mathrm{Cu})$ nanofluid is considered in this study. The model used for the nanofluid incorporates the effect of the nanoparticles volume fraction. The governing partial differential equations are transformed into ordinary differential equations by similarity transformations. The transformed equations are solved numerically by using a shooting method. Results for the skin friction coefficient, local Nusselt number, velocity profiles and temperature profiles are presented for different values of the governing parameters. The analysis reveals the conditions for the existence of the steady boundary layer flow due to shrinking of the sheet and it is found that when the mass suction parameter exceeds a certain critical value, steady flow is possible. Dual solutions for the velocity and temperature distributions are obtained. With increasing values of the nanoparticles volume fraction, the skin friction and the heat transfer coefficient increase.
\end{abstract}

Keyword: Boundary layer; Dual solutions; Heat transfer; Nanofluid; Shrinking sheet 